\title{
Analysis and comprehensive comparison of PacBio and nanopore-based RNA sequencing of the Arabidopsis transcriptome
}

Jiawen Cui

Yangzhou University

Nan Shen

Yangzhou University

Zhaogeng Lu

Yangzhou University

Guolu Xu

Biomarker Technologies Corporation

Yuyao Wang

Biomarker Technologies Corporation

Biao Jin ( $\nabla$ bjin@yzu.edu.cn )

Yangzhou University https://orcid.org/0000-0002-7330-334X

\section{Methodology}

Keywords: Plant transcriptome, Third-generation sequencing, PacBio, Nanopore, RNA-Seq, Singlemolecule sequencing

Posted Date: December 19th, 2019

DOI: https://doi.org/10.21203/rs.2.19252/v1

License: (c) (i) This work is licensed under a Creative Commons Attribution 4.0 International License.

Read Full License

Version of Record: A version of this preprint was published at Plant Methods on June 12th, 2020. See the published version at https://doi.org/10.1186/s13007-020-00629-x. 


\section{Abstract}

Background The number of studies using third-generation sequencing using Pacific Biosciences (PacBio) and Oxford Nanopore Technologies (ONT) is rapidly increasing in many different research areas. Among them, plant full-length single-molecule transcriptome studies have mostly used PacBio sequencing, whereas ONT is rarely used. Therefore, in this study, we examined ONT RNA sequencing methods in plants. We performed a detailed evaluation of reads from PacBio, Nanopore direct cDNA (ONT Dc), and Nanopore PCR CDNA (ONT PC) sequencing including characteristics of raw data and identification of transcripts. In addition, matched Illumina data were generated for comparison.

Results ONT Pc showed overall better raw data quality, whereas PacBio generated longer read lengths. In the transcriptome analysis, PacBio and ONT Pc performed similarly in transcript identification, simple sequence repeat analysis, and long non-coding RNA prediction. PacBio was superior in identifying alternative splicing events, whereas ONT Pc could estimate transcript expression levels.

Conclusions This paper made a comprehensive comparison of PacBio and nanopore-based RNA sequencing of the Arabidopsis transcriptome, the results indicate that ONT Pc is more cost-effective for generating extremely long reads and can characterise the transcriptome as well as quantify transcript expression. Therefore, ONT Pc is a new cost-effective and worthwhile method for full-length singlemolecule transcriptome analysis in plants.

\section{Background}

Current sequencing-based transcriptomic analyses (RNA-Seq) using the massive throughput of nextgeneration sequencing platforms have enabled us to build a picture of the active transcriptional patterns within organisms. Among these analyses, short-read RNA-Seq (mainly using Illumina technology) has been used for over a decade. The numbers of reads output by Illumina sequencers are sufficient to accurately quantify gene expression. However, because Illumina sequencers are appropriate only for short read-length sequencing, we must fragment RNA or cDNA during sample preparation. Thus, RNA transcript information covering long lengths or distances, including alternative splicing (AS), simple sequence repeats (SSRs), and long non-coding RNA (IncRNA), is lost using this technology.

With the rapid development of sequencing technology, long-read sequencing platforms, including Pacific Biosciences (PacBio) [1] and Oxford Nanopore Technologies (ONT) [2], which have the capability to sequence entire cDNA molecules end-to-end, are available. These two platforms have increased read lengths considerably in comparison to next-generation sequencing methods and can be used to address a large variety of research questions. PacBio single-molecule real-time (SMRT) isoform sequencing (IsoSeq) can capture the full length of transcripts, thereby presenting an easier and more accurate method for gene annotation [3], isoform identification [4, 5], and IncRNA discovery [6]. Thus, it has been successively used for whole-transcriptome profiling in many different organisms [7-9]. On the other hand, ONT sequencers measure changes in ionic current when the DNA fragments translocate through protein 
nanopores in a semi-synthetic insulated membrane; this process does not require enzyme-based nucleotide incorporation or detection of fluorescence signals. Therefore, the sequencing read length is theoretically limited only by the length of the DNA fragment translocating through the pore, and amplification biases can be avoided. For these reasons, ONT sequencing has recently allowed researchers to produce high-quality whole-genome assemblies of species such as humans [10], Arabidopsis thaliana [11], and Solanum pennellii [12]. Increasing studies are utilising ONT sequencers for transcriptome sequencing in humans and animals; however, studies of plants are limited.

In this study, we conducted a comprehensive comparison of PacBio and ONT applications in plant transcriptome sequencing, including read length, error rate, error pattern, coding region (CDS) and IncRNA prediction, complex transcriptome event discovery, and transcript abundance using ONT. This work provides a valuable reference for applications of ONT in plant transcriptome analysis.

\section{Results}

\section{Overview of Illumina, PacBio, and ONT sequencing}

To compare the performance of the RNA sequencing methods, we sequenced cDNA libraries from Arabidopsis on Illumina NovaSeq, PacBio Sequel, and Nanopore GridION instruments. In addition, using Oxford nanopore sequencing, we sequenced cDNA directly (ONT Dc) and amplified cDNA (ONT Pc).

After sequencing, we obtained more than 21 million clean reads from each Illumina RNA-Seq replicate (Table S1). The clean reads of each replicate were mapped to the reference genome; the percent of total mapped reads was $>84.67 \%$ (Table S2).

For PacBio SMRT sequencing, one size-fractionated, full-length cDNA library (1-6 kb) was constructed and subsequently sequenced in one SMRT cell. As a result, we obtained $26.71 \mathrm{~Gb}$ of clean data. With full passes $\geq 0.8$ and a predicted consensus accuracy $>0.80,516,364$ ROIs were successfully extracted with 27 passes and with a mean length of $1799 \mathrm{bp}$ and quality of 0.97 (Table S3). These ROls included 416,662 (80.7\%) full-length non-chimeric (FLNC) and 79,984 (15.5\%) non-full-length (nFL) reads (Fig. 1). Then, using the ICE algorithm for clustering, we finally obtained 181,135 consensus isoforms in Arabidopsis.

For ONT Dc sequencing, we obtained 6,892,169, 5,687,972, and 10,936,056 clean reads from CTRL1, CTRL2, and CTRL3, respectively. The N50 values of these reads were 1245,1438 , and 1345, and the mean lengths were 1065,1228 , and 1167 , respectively. Then, full-length sequences were identified if primers were found at both ends. As a result, we obtained 128,781, 138,295, and 262,832 full-length reads (FL reads) from CTRL1, CTRL2, and CTRL3, respectively (Table S4). For ONT Pc sequencing, 8,146,264, $7,713,840$, and 6,912,956 clean reads were obtained from CTRL1, CTRL2, and CTRL3, respectively. The N50 values of these reads were 1252, 1292, and 1270 and the mean lengths were 1222, 1246, and 1225, respectively. Among them, 5,682,227, 5,563,209, and 5,207,164 FL reads were obtained from CTRL1, CTRL2, and CTRL3, respectively (Table S4). 


\section{Comparison of raw data from PacBio and ONT results}

To compare the raw data from PacBio and ONT sequencing, we randomly selected $10 \mathrm{Mb}$ raw reads $(3,112,439$ subreads) from PacBio and 100,000 1D reads from each ONT sample (300,000 total).

The read length is a good representation of the useful length of long reads. The mean length of PacBio reads was $1410.186 \mathrm{bp}$, with median and maximum read lengths of $1302 \mathrm{bp}$ and 89,075 bp respectively. ONT Dc data were shorter, with median and maximum lengths of $771 \mathrm{bp}$ and $61,315 \mathrm{bp}$, respectively. The median and maximum read lengths of ONT Pc data were 1097 bp and 8236 bp, respectively (Table 1).

The overall length distributions of the reads for both PacBio and ONT exhibited remarkable differences (Fig. $2 \mathrm{a}-\mathrm{c}$ ). Compared to PacBio, the length distribution of ONT Dc data was skewed to the left, with a large proportion of reads $<2000 \mathrm{bp}$ in length (Fig. $2 a-c$ ). In addition, the length distribution of ONT Pc data was similar to that of the ONT Dc data (Fig. 2b, c).

Mappability of long reads is essential for confirming repetitive elements, gene isoforms, and gene fusions. Of PacBio subreads, 94.5\% were aligned to the reference genome (Fig. 2d, Table 2). Compared to the PacBio subreads, ONT Dc 1D reads had a lower rate (66\%) (Fig. 2e, Table 2), and ONT Pc 1D reads had a higher rate of alignment (97\%) (Fig. 2f, Table 2). For PacBio and ONT Pc data, we found that short read lengths $(<500 \mathrm{bp}$ ) had low alignment rates (Fig. 2d, f). This is likely due to a larger portion of adapter and linker sequences in this short-length data bin. However, of the ONT Dc 1D reads, all lengths had similar alignment rates (around 60\%) (Fig. 2e).

Some regions of long reads may be particularly error prone, and long reads may be aligned as separated fragments, called gap-aligned reads. Corresponding to the high error rate, more ONT Dc data were gapped alignments $(0.08 \%)$ compared to PacBio subreads $(0.02 \%)$ and ONT Pc 1D reads $(0.02 \%)$ (Table 2 ). These rates are very low and can be considered negligible in third-generation sequencing data. Long reads generated from gene fusions or trans-splices can be aligned to separate genomic loci; these are termed "trans-chimeric reads". PacBio subreads contained $0.74 \%$ trans-chimeric reads, whereas ONT 1D data contained fewer (ONT Dc: $0.24 \%$ and ONT Pc: $0.43 \%$ ) (Table 2). In addition, the PacBio subreads showed notably higher trans-chimeric rates in very long reads ( $>4 \mathrm{~kb})$ (Fig. 2d). Two fragments of a long read may be aligned to the same genomic locus, termed "self-chimeric", because of the failure to remove adaptor sequences from the raw data. PacBio subreads and ONT Pc 1D reads contained $0.46 \%$ and $0.08 \%$ self-chimeric reads, respectively, while ONT Dc 1D reads had a surprisingly higher rate $(4.89 \%)$ (Table 2). The chimeric reads may cause overestimation of DNA molecule lengths.

Error rates and error patterns can indicate the quality of the data, which has a strong effect on singlenucleotide resolution analysis. The error rate of PacBio was $13.217 \%$. Compared to the PacBio subreads, the error rate of ONT Dc data was slightly higher, reaching $13.934 \%$, while the error rate of ONT Pc data was lower (12.669\%) (Table 3). This indicated that the ONT Pc data were of slightly higher base quality than PacBio data. 
In addition, the compositions of PacBio and ONT errors were similar. The proportions of mismatches were $4.084 \%, 4.710 \%$, and $4.352 \%$ for PacBio, ONT Dc, and ONT Pc data, respectively (Table 3). The deletions had higher rates, 5.205\%, 5.851\%, and 5.085\%, in PacBio, ONT Dc, and ONT Pc data, respectively; the insertion rates were $3.928 \%, 3.374 \%$, and $3.232 \%$. Taken together, the deletions and insertions together (indels) contributed the most errors in both PacBio and ONT data.

Both PacBio and ONT errors exhibited context-specific patterns. In PacBio reads, most mismatches arose from several context-specific events such as $G A \rightarrow T A, G C \rightarrow T C, G G \rightarrow T G$, and GT $\rightarrow T T$ (Fig. 3a). The mismatch $C G \rightarrow C A$ was most abundant in ONT Dc data, followed by AG $\rightarrow$ GG (Fig. 3b). The contextspecific mismatches in ONT Pc data were similar to those in ONT Dc data, with CG $\rightarrow$ CA as the most abundant (Fig. 3c). In addition, $T$ in insertions and $A$ and $G$ in deletions were most commonly observed in PacBio reads (Fig. 3a), whereas $A$ in insertions and $A$ and $C$ in deletions were most common in ONT data (Fig. 3b, c).

\section{Transcriptome construction from Illumina, PacBio, and ONT data}

Based on Illumina short reads, a total of 21,157 genes were obtained. For PacBio sequencing, the consensus isoforms were polished using non-full-length reads, and 129,080 transcripts with high quality were obtained. These corrected transcripts were mapped to the reference genome using GMAP software. After removal of redundant reads, 38,011 non-redundant mapped transcripts from 13,376 gene loci were generated. For ONT Dc sequencing, after polishing all of the full-length reads, the corrected isoforms were mapped against the reference genome, generating a total of 47,601 unique mapped transcripts. Similarly, ONT Pc sequencing generated 36,775 non-redundant mapped transcripts.

We first compared the transcript lengths among the different sequencing technologies. The mean length of PacBio transcripts was 2072.57 bp; the median and maximum read lengths were $1988 \mathrm{bp}$ and 8105, respectively. ONT Dc data were shorter, with median and maximum lengths of $1249.97 \mathrm{bp}$ and $6763 \mathrm{bp}$. The median and maximum read lengths of ONT Pc data were $1332 \mathrm{bp}$ and $7319 \mathrm{bp}$, respectively (Table 4).

\section{AS events}

Within the PacBio unique mapped reads, we detected a total of 12,979 AS events, including 97 mutually exclusive exon events, 8,175 intron retention (IR) events, 611 exon-skipping (ES) events, 1,430 alternative $5^{\prime}$ sites (Alt. 5'), and 2,666 alternative $3^{\prime}$ sites (Alt. $3^{\prime}$ ). In PacBio data, the most frequent AS events identified were IR events (62.99\%), followed by Alt. 3' (20.54\%), Alt. 5' (11.02\%), and ES events (4.71\%); few mutually exclusive exon events (0.75\%) were discovered (Fig. 4a).

Far fewer AS events were detected in ONT Dc data; CTRL1, CTRL2, and CTRL3 contained 1433, 928, and 4367 AS events, respectively. The fractions of each AS type also differed from the PacBio data. The most identified AS events in ONT data were Alt. $3^{\prime}$ events, followed by Alt. 5', IR, and mutually exclusive exon events; the fewest were ES events (1.07\%) (Fig. 4b). By contrast, in the ONT Pc data, 1897, 2048, and 
2034 AS events were identified in CTRL1, CTRL2, and CTRL3, respectively. The fractions of each AS type were similar to those of the PacBio data (Fig. 4c).

\section{SSR detection}

Transcripts $>500$ bp in length were selected for SSR analysis using MISA. A total of 58,885 sequences $(122,942,629 \mathrm{bp})$ were subjected to SSR analysis. As a result, we identified a total of 29,394 SSRs and 20,243 SS-containing sequences from PacBio data (Table S5). There were 6067 sequences containing more than one SSR, and 4,109 SSRs were present in compound formation. Furthermore, the numbers of mononucleotide, di-nucleotide, tri-nucleotide, tetra-nucleotide, penta-nucleotide, and hexa-nucleotide SSRs were $15,353,5,377,8,507,89,9$, and 59 , respectively.

Within ONT Dc data, 32,854 sequences of $>500$ bp $(53,964,961)$ were used for SSR analysis. A total of 9,234 SSRs and 7,543 SSR-containing sequences were identified. Similarly, in the ONT Pc data, 35,305 transcripts $(53,588,806$ bp) contained 13,415 SSRs and 10,350 SSR-containing sequences (Table S5).

\section{CDSs of new transcripts and IncRNA prediction}

Using TransDecoder (v3.0.0), 31,137 ORFs were identified in the PacBio data, of which 25,256 were complete ORFs. In addition, 33,419 and 15,968 complete ORFs were predicted in the ONT Dc and ONT Pc data, respectively. Figure 5 a shows the length distribution of the CDSs of complete ORFs. In the PacBio data, the CDS lengths of complete ORFs mostly ranged from 100 to $1000 \mathrm{bp}$ (Fig. 5a). However, the length distribution was skewed to the left in the ONT Dc and ONT Pc data. In the ONT Dc data, most CDS lengths of complete ORFs were $0-100 \mathrm{bp}$, followed by 100-200 bp, and only a few reads were $>200 \mathrm{bp}$. Similarly, in the ONT Pc data, the lengths of CDS of complete ORFs ranged from 0 to $800 \mathrm{bp}$, with most being $0-300$ bp (Fig. 5a).

Using CPC, $\mathrm{CNCl}$, Pfam protein structure domain analysis, and CPAT, totals of 257, 8911, and 249 IncRNAs were predicted by all four methods from PacBio, ONT Dc, and ONT Pc data, respectively (Fig. $5 b-d)$. Thirty-five common IncRNAs were identified both in PacBio and ONT Pc data (Table S6). We randomly selected 16 unique IncRNAs (8 IncRNAs from PacBio data and 8 IncRNAs from ONT Pc data) for validation by PCR amplification and Sanger sequencing. Of the 8 IncRNAs from PacBio data, 2 were completely identical to the RNA-Seq sequences, and 2 had fewer than three mismatched nucleotides (Fig. 6). In addition, of the eight selected IncRNAs from ONT Pc data, 5 IncRNAs were verified, all of which had fewer than three mismatched nucleotides.

\section{Isoform abundance estimation by ONT and Illumina data}

We evaluated the performances of ONT and Illumina data on transcript quantification. Fragments per kilobase of transcript per million fragments mapped (FPKM), reads per transcript per 10,000 reads (RPT10K) values and counts per million (CPM) values were used to quantify transcript expression levels of Illumina, ONT Dc and ONT Pc data, respectively. We further calculated the correlation between Illumina and ONT Dc data of each repeat. The results showed that the expression correlation values between 
Illumina and ONT data of CTRL1, CTRL2, and CTRL3 were $0.781,0.765$, and 0.777 , respectively (Fig. 7ac). The expression correlation values between Illumina and ONT Pc were higher, at 0.821, 0.855, and 0.838 for CTRL1, CTRL2, and CTRL3, respectively (Fig. 7d-f).

\section{Discussion}

Next-generation sequencing is still the primary technology used for transcriptome studies. However, shortread RNA-Seq methods are limited in their ability to identify complex transcript isoforms because they cannot sequence full-length transcripts. Furthermore, transcripts are fragmented, which results in short individual reads that fail to span the entirety of the transcripts. To overcome the limitations of short-read RNA-Seq, TGS, including single-molecule long-read PacBio Iso-Seq and ONT, was developed. To date, many studies have successfully used PacBio Iso-Seq to sequence full-length cDNA samples derived from both animals and plants $[7,13]$. In recent years, ONT has also been used to analyse full-length CDNA samples from mouse individual B cells [14] and human embryonic stem cells [15]. In practice, PacBio and ONT sequencing have their own merits and demerits. Briefly, PacBio sequencers produce low numbers of reads with high accuracy, while ONT sequencers produce higher numbers of reads with lower accuracy. Furthermore, PacBio Iso-Seq is commercially expensive, and ONT sequencing is more cost-effective. Thus, in the present study, we performed a detailed evaluation of reads from PacBio and Nanopore direct CDNA and PCR CDNA sequencing in plants (Arabidopsis) based on the characteristics of raw data and identification of transcripts. In addition, matched Illumina data were generated for comparison of transcript expression between ONT and Illumina. We aimed to select a method to obtain long stretches of sequences at bargain basement prices.

Overall, we observed that PacBio and ONT can similarly generate long read lengths with relatively high error rates. However, they have some differences in their raw data. Because the quality of ONT DC sequencing was far below that of ONT Pc sequencing, we focused on the comparison between the results of PacBio and ONT Pc sequencing. First, among the randomly selected raw data, the maximum read length of PacBio results was $89,075 \mathrm{bp}$, whereas that of ONT Dc data was $61,315 \mathrm{bp}$. Unexpectedly, the maximum read length of ONT Pc data was notably shorter than those of PacBio and ONT Dc, at only $8,236 \mathrm{bp}$. Second, previous studies have shown that PacBio can generate higher quality raw data with a lower error rate and higher mappability compared to ONT raw data [15]. In our study, the mappability of ONT Pc data was $97.0 \%$, whereas that of PacBio raw data was $94.5 \%$. In addition, the error rate of PacBio was $13.217 \%$, and the ONT error rate was slightly lower at $12.669 \%$. Thus, our results indicated that ONT Pc data were of a higher quality, suggesting that ONT may be a promising tool for transcriptome-wide studies.

TGS methods have been applied in sequencing of animals and humans and have proven superior to short-read sequencing methods due to the advantage of obtaining full-length transcripts [16]. Thus, they can be used to identify complex AS events, which can greatly increase transcriptome diversity. AS events include five different types: mutually exclusive exons, IR, ES, Alt. 5', and Alt. 3'. Many studies have used long reads from PacBio and ONT to detect AS events. For example, Iso-Seq identified 10,053 and 21,154 
AS events in Sorghum bicolor [4] and Phyllostachys edulis [5], respectively. In our study, PacBio identified 12,979 AS events, whereas ONT Pc only identified 2,000 AS events from each sample. The number of AS events identified was much higher in the PacBio data, indicating that PacBio sequencing results may serve as a more valuable resource for the study of transcriptome complexity and gene regulation.

LncRNAs are defined as RNAs that are $>200 \mathrm{nt}$ in length and have no coding potential [17]. Most IncRNAs are polyadenylated in plants, so Illumina RNA-Seq can both detect IncRNAs and quantify their expression. However, recently, several studies have reported that IncRNAs undergo complex post-transcriptional regulation [18], and full-length sequencing showed great advantages in identifying gene models of IncRNAs. Iso-Seq in poplar and Trifolium pratense L. revealed 1,187 and 4,333 IncRNAs, respectively [19, 20], suggesting that Iso-Seq is a suitable method for identification of IncRNAs. Here, we identified 257 and 249 IncRNAs based on PacBio and ONT Pc data. Furthermore, PCR and Sanger sequencing validated four of eight and five of eight IncRNAs from the PacBio and ONT Pc data, respectively. These results suggest that both ONT Pc and PacBio methods are suitable for identification of IncRNAs.

Compared to PacBio sequencing, one of the greatest advantages of ONT is that it can estimate transcript expression levels [21]. In the present study, we analysed the correlation between Illumina and ONT DC data of each replicate sample and found correlations $>0.8$ for all groups. The high correlation suggests that ONT can well quantify transcript expression levels.

\section{Conclusions}

In conclusion, our results showed that ONT Pc performed well in transcript identification, SSR analysis, and IncRNA prediction. ONT Pc generated better quality data in terms of error rate and mappability, and PacBio generated longer sequence reads. Although PacBio is superior in identifying AS events, ONT Pc can quantify transcripts of different lengths. In addition, ONT is less expensive than PacBio. Taken together, these results indicate that ONT Pc is more cost-effective for generating extremely long reads and can characterise the transcriptome as well as quantify transcript expression. Therefore, it is a good choice for full-length single-molecule transcriptome analysis in plants.

\section{Methods}

Seeds of $A$. thaliana [wild-type Columbia (Col-0)] were exposed to stratification for $2 \mathrm{~d}$ at $4^{\circ} \mathrm{C}$, then sown in square surface-sterilised plastic pots $(7 \mathrm{~cm} \times 7 \mathrm{~cm} \times 8 \mathrm{~cm})$ containing sterile medium [1:1 (v/v) mixture of vermiculite and peat]. Pots were arranged in a plastic pallet and placed in a growth chamber $\left(23^{\circ} \mathrm{C}\right.$ during the day and $18^{\circ} \mathrm{C}$ at night, with a 16 -h photoperiod and $500 \mu \mathrm{mol} \mathrm{m}^{-2} \mathrm{~s}^{-1}$ of photosynthetically active radiation); the plants were watered to saturation with, alternately, distilled water or $1 / 2$ MurashigeSkoog solution [22]. The aboveground parts were collected at a growth stage of 3.90 (rosette growth complete). Each replication contained 15-20 plants. 
Total RNA was extracted using the RNAprep Pure Plant Kit (Tiangen, Beijing, China). The RNA Nano 6000 Assay Kit of the Agilent Bioanalyzer 2100 system (Agilent Technologies, Santa Clara, CA, USA) was used to assess RNA integrity, and the Qubit RNA Assay Kit and Qubit 2.0 Fluorometer (Life Technologies, Carlsbad, CA, USA) were used to quantify the extracted RNA.

\section{Library preparation and sequencing}

For Illumina sequencing, cDNA libraries (with three biological replicates) were constructed using the NEBNext Ultra RNA Library Prep Kit for Illumina (New England Biolabs, Beverly, MA, USA) following the manufacturer's protocol. The libraries were sequenced on an Illumina NovaSeq platform, and paired-end reads were generated.

For PacBio sequencing, cDNA from the same RNA samples used for Illumina sequencing was synthesised using the SMARTer PCR cDNA Synthesis Kit. After PCR amplification, products were sequenced on the PacBio Sequel platform.

For ONT sequencing, CDNA-PCR libraries were built using the Ligation Sequencing Kit (SQK-LSK109) and sequenced on the Nanopore PromethION platform. Direct-cDNA libraries were built using the Direct cDNA Sequencing Kit (SQK-DCS108) and sequenced on the Nanopore GridION X5 platform.

\section{Comprehensive quality assessment for long-read sequencing}

The AlignQC software (https://github.com/jason-weirather/AlignQC/wiki) was used to perform the comprehensive quality assessment for long-read sequencing. First, all data were uploaded into the software, and the memory was adjusted to $500 \mathrm{~GB}$, however, the memory was insufficient. Therefore, we randomly selected $10 \mathrm{Mb}$ raw reads from the PacBio results and 100,000 1D reads from each ONT sample for subsequent analyses. AlignQC accepted standard BAM format files as inputs and output XHTML format files for easy visualisation, providing links to access all analysis results.

\section{Transcript identification of PacBio long reads}

The SMRT-Analysis software package v3.0 (https://github.com/ben-lerch/IsoSeq3.0/blob/master/README.md) was used for Iso-Seq data analysis. First, reads of insert (ROIs) were generated using full passes $\geq 0.8$. Then, with the examination of poly $(A)$ signals and $5^{\prime}$ and $3^{\prime}$ adaptors, full-length and non-full-length CDNA reads were recognised. Consensus isoforms were identified by iterative clustering for error correction (ICE) algorithm and further polished to obtain high-quality consensus isoforms. Then the high-quality isoforms were mapped to the reference genome of Arabidopsis. Redundancy was removed from the mapped results using cDNA_Cupcake (https://github.com/Magdoll/cDNA_Cupcake/wiki). The reads with identity $<0.9$ or coverage $<0.85$ were filtered out, and reads in which only the 5 -end exons differed were combined. As a result, non-redundant transcripts were obtained.

\section{Transcript identification of ONT long reads}


Nanopore sequencing raw data were base-called using the Guppy software in MinKNOW2.2. Then, short reads, low-quality reads, and reads with adaptors were filtered out to obtain the clean data. According to the principle of cDNA sequencing, a primer sequence identified at both ends of a read was indicative of a full-length sequence. Then, using minmap2 to map the read itself, overlap information between reads was obtained. Finally, consistent sequences were obtained using the Racon software. The high-quality isoforms were mapped to the reference genome of Arabidopsis using the Genomic Mapping and Alignment Program (GMAP). Redundancy was removed from the mapped results by same method used in the PacBio data analysis.

\section{SSR detection}

Transcripts larger than 500 bp were selected for SSR analysis using the MIcroSAtellite identification tool (MISA). MISA can identify seven SSR types, including mono-nucleotide, di-nucleotide, tri-nucleotide, tetranucleotide, penta-nucleotide, hexa-nucleotide, and compound SSRs.

\section{Coding sequence prediction of new genes}

The coding sequences and corresponding amino acid sequences within the transcript sequences were predicted by TransDecoder. TransDecoder can identify candidate protein-coding regions based on nucleotide composition, open reading frame (ORF) length, log-likelihood score, and optional protein family database (Pfam) domain content.

\section{IncRNA prediction}

The transcripts with coding potential were filtered by the Coding Potential Calculator (CPC), Coding-NonCoding Index (CNCl), Coding Potential Assessment Tool (CPAT), and Pfam. Finally, the remaining noncoding transcripts were selected as IncRNAs.

\section{Cloning and Sanger sequencing of IncRNAs}

Total RNA was isolated from Arabidopsis as described above. cDNA was synthesised from $2 \mu \mathrm{g}$ of purified total RNA using the PrimeScript 1st Strand cDNA Synthesis Kit (TaKaRa, Dalian, China) according to the manufacturer's protocol. Sixteen pairs of primers were designed (Table S7). PCR amplifications were carried out as follows: $94^{\circ} \mathrm{C}$ for $5 \mathrm{~min}$, followed by 35 cycles at $94^{\circ} \mathrm{C}$ for $35 \mathrm{~s}, 55^{\circ} \mathrm{C}$ or $58^{\circ} \mathrm{C}$ for $15 \mathrm{~s}$, and $72^{\circ} \mathrm{C}$ for $50 \mathrm{~s}$. Amplification products were separated on a $2 \%$ agarose gel. Gel-purified PCR fragments were cloned into the T5-simple Vector system (TransGen, Beijing, China) and sequenced.

\section{Quantification of gene expression levels}

For Illumina sequencing, gene expression levels were estimated by fragments per kilobase of transcript per million fragments mapped (FPKM) using Cufflinks software (23). For ONT Pc sequencing, gene expression levels were estimated by counts per million (CPM) (24). The formula is shown as follow: CPM $=($ reads mapped to transcript/total reads aligned in sample) ' $1,000,000$. For ONT Dc sequencing, gene 
expression levels were estimated by reads per gene/transcript per 10,000 reads mapped (RPT10K) (25). The formula is shown as follow: RPT10K = (reads mapped to transcript/total reads aligned in sample) ' 10000.

\section{Declarations}

\section{Acknowledgements}

We thank Biomarker Technologies for helping with RNA-seq by Illumina, PacBio and ONT technologies.

\section{Author Contributions}

JWC and BJ participated in the design of the research and drafted the manuscript. JWC, NS and ZGL performed the experimental work and contributed the data analysis, JWC participated in the sampling. GLX and YYW contributed the sequencing and data computing optimization. All authors read and approved the final manuscript.

\section{Funding}

This work was supported by self-funding.

\section{Availability of data and materials}

All raw sequence data have been deposited in the NCBI GSE 141641

(https://www.ncbi.nlm.nih.gov/geo/query/acc.cgi?acc=GSE141641).

\section{Ethics approval and consent to participate}

Not applicable.

\section{Consent for publication}

Not applicable.

\section{Competing interests}

The authors declare that they have no competing interests

\section{Author details}

${ }^{1}$ College of Horticulture and Plant Protection, Yangzhou University, Yangzhou 225009, China.

${ }^{2}$ Biomarker Technologies Corporation, Beijing, 101300, China

\section{References}


1. Rhoads A, Au KF. PacBio sequencing and its applications. Genom Proteome Bioinf. 2015; 13(5): 278289.

2. Bayega A, Fahiminiya S, Oikonomopoulos S, Ragoussis J. Current and future methods for mRNA analysis: a drive toward single molecule sequencing//Gene Expression Analysis. New York: Humana Press; 2018. p. 209-241.

3. Gonzalez-Garay ML. Introduction to isoform sequencing using pacific biosciences technology (IsoSeq)//Transcriptomics and Gene Regulation. Dordrecht: Springer; 2016. p. 141-160.

4. Abdel-Ghany SE, Hamilton M, Jacobi JL, Ngam P, Devitt N, Schilkey F, Ben-Hur A, Reddy ASN. A survey of the sorghum transcriptome using single-molecule long reads. Nat Commun. 2016; 7: 11706.

5. Wang T, Wang H, Cai D, Gao Y, Zhang H, Wang Y, Lin C, Ma L, Gu L. Comprehensive profiling of rhizome-associated alternative splicing and alternative polyadenylation in moso bamboo (Phyllostachys edulis). Plant J. 2017; 91(4): 684-699.

6. Li S, Yamada M, Han X, Ohler Uwe, Benfey PN. High-resolution expression map of the Arabidopsis root reveals alternative splicing and lincRNA regulation. Dev Cell. 2016; 39(4): 508-522.

7. Sharon $D$, Tilgner $H$, Grubert $F$, Snyder M. A single-molecule long-read survey of the human transcriptome. Nat Biotechnol. 2013; 31(11): 1009.

8. Thomas S, Underwood J G, Tseng E, Holloway AK. Long-read sequencing of chicken transcripts and identification of new transcript isoforms. Plos One. 2014; 9(4): e94650.

9. Wang B, Tseng E, Regulski M, Clark TA, Hon T, Jiao Y, Lu Z, Olson A, Stein JC, Ware D. Unveiling the complexity of the maize transcriptome by single-molecule long-read sequencing. Nat Commun. 2016; 7: 11708 .

10. Jain M, Koren S, Miga KH, Quick J, Rand A, Sasani TA, Tyson JR, Beggs AD et al. Nanopore sequencing and assembly of a human genome with ultra-long reads. Nat Biotech. 2018; 36(4): 338.

11. Michael TP, Jupe F, Bemm F, Motley ST, Sandoval JP, Lanz C, Loudet O, Weigel D, Ecker JR. High contiguity Arabidopsis thaliana genome assembly with a single nanopore flow cell. Nat Commun. 2018; 9(1): 541.

12. Schmidt MHW, Vogel A, Denton AK, Istace B, Wormit A, Geest H, Bolger ME, Alseekh S, Maß J, Pfaff C, Schurr U, Chetelat R, Maumus F, Aury JM, Koren S, Fernie AR, Zamir D, Bolger AM, Usadel B. De novo assembly of a new Solanum pennellii accession using nanopore sequencing. Plant Cell. 2017; 29(10): 2336-2348.

13. Treutlein B, Gokce O, Quake SR, Südhof TC. Cartography of neurexin alternative splicing mapped by single-molecule long-read mRNA sequencing. P Natl Acad Sci, 2014; 111(13): E1291-E1299.

14. Byrne A, Beaudin AE, Olsen HE, Jain M, Cole C, Palmer T, DuBois RM, Forsberg EC, Akeson M, Vollmers $\mathrm{C}$. Nanopore long-read RNAseq reveals widespread transcriptional variation among the surface receptors of individual B cells. Nat Commun. 2017; 8: 16027.

15. Weirather JL, de Cesare M, Wang Y, Piazza P, Sebastiano V, Wang XJ, Buck D, Au K. Comprehensive comparison of Pacific Biosciences and Oxford Nanopore Technologies and their applications to 
transcriptome analysis. F1000Res. 2017; 6: 100.

16. Wang Z, Zhang H, Gong W. Genome-wide identification and comparative analysis of alternative splicing across four legume species. Planta. 2019; 249(4): 1133-1142.

17. Laurent GS, Wahlestedt C, Kapranov P. The Landscape of long noncoding RNA classification. Trends Genet. 2015; 31(5): 239-251.

18. Liu J, Wang H, Chua NH. Long noncoding RNA transcriptome of plants. Plant Biotechnol J. 2015; 13(3): 319-328.

19. Chao Q, Gao ZF, Zhang D, Zhao BG, Dong FQ, Fu C, Liu L, Wang B. The developmental dynamics of the Populus stem transcriptome. Plant Biotechnol J. 2019; 17(1): 206-219.

20. Chao Y, Yuan J, Li S, Jia S, Han S, Xu L. Analysis of transcripts and splice isoforms in red clover (Trifolium pratense) by single-molecule long-read sequencing. BMC Plant Biol. 2018; 18(1): 300.

21. Seki M, Katsumata E, Suzuki A, Sereewattanawoot S, Sakamoto Y, Mizushima-Sugano J, Sugano S, Kohno T, Frith MC, Tsuchihara K. Evaluation and application of RNA-Seq by MinION. DNA Res. 2018; 26(1): 55-65.

22. Jin B, Wang L, Wang J, Jiang KZ, Wang Y, Jiao XX, Ni CY, Wang YL, Teng NJ. The effect of experimental warming on leaf functional traits, leaf structure and leaf biochemistry in Arabidopsis thaliana. BMC Plant Biol. 2011; 11(1): 35.

23. Florea L, Song L, Salzberg SL. Thousands of exon skipping events differentiate among splicing patterns in sixteen human tissues. F1000Res. 2013; 2:188.

24. Zhou $X$, Lindsay $H$, Robinson MD. Robustly detecting differential expression in RNA sequencing data using observation weights. Nucleic Acids Res. 2014; 42(11): e91-e91.

25. Byrne A, Beaudin AE, Olsen HE, Jain M, Cole C, Palmer T, DuBois RM, Forsberg EC, Akeson M, Vollmers $\mathrm{C}$. Nanopore long-read RNAseq reveals widespread transcriptional variation among the surface receptors of individual B cells. Nat Commun. 2017; 8: 16027.

\section{Tables}

Table 1. Read length distribution of PacBio and ONT raw data

\begin{tabular}{|c|c|c|c|}
\hline \multirow{2}{*}{ Sequencing type } & \multicolumn{3}{|c|}{ Read length } \\
\cline { 2 - 4 } & Mean & median & maximum \\
\hline PacBio & 1410.186 & 1302 & 89075 \\
\hline ONT Dc & 902.0619 & 771 & 61315 \\
\hline ONT Pc & 1231.308 & 1097 & 8236 \\
\hline
\end{tabular}

Table 2 Alignment results of PacBio and ONT data 


\begin{tabular}{|l|l|l|l|}
\hline & PacBio & ONT Dc & ONT Pc \\
\hline Total reads & $3,112,439$ & 300,000 & 300,000 \\
\hline Unaligned reads & $170,385(5.5 \%)$ & $101,892(34.0 \%)$ & $8,909(3.0 \%)$ \\
\hline Aligned reads & $2,942,054(94.5 \%)$ & $198,108(66.0 \%)$ & $291,091(97.0 \%)$ \\
\hline Single-align reads & $2,904,280(93.3 \%)$ & $182,478(60.8 \%)$ & $289,504(96.5 \%)$ \\
\hline Gapped-align reads & $529(0.02 \%)$ & $237(0.08 \%)$ & $50(0.02 \%)$ \\
\hline Chimeric reads & $37,245(1.20 \%)$ & $15,393(5.13 \%)$ & $1,537(0.51 \%)$ \\
\hline Trans-chimeric reads & $22,921(0.74 \%)$ & $710(0.24 \%)$ & $1,296(0.43 \%)$ \\
\hline Self-chimeric reads & $14,324(0.46 \%)$ & $14,683(4.89 \%)$ & $241(0.08 \%)$ \\
\hline
\end{tabular}

Table 3 Error pattern of PacBio and ONT data

\begin{tabular}{|l|l|l|l|}
\hline & PacBio & ONT Dc & ONT PC \\
\hline Bases analyzed & $1,117,976$ & $1,066,943$ & $1,079,480$ \\
\hline Correctly aligned bases & $970,213(86.8 \%)$ & $917,270(86.1 \%)$ & $942,722(87.3 \%)$ \\
\hline Total error bases & $147,7963(13.217 \%)$ & $148,673(13.934 \%)$ & $136,758(12.669 \%)$ \\
\hline Mismatched bases & $45,654(4.084 \%)$ & $50,249(4.710 \%)$ & $46,978(4.352 \%)$ \\
\hline Deletion bases & $58,194(5.205 \%)$ & $62,423(5.851 \%)$ & $54,896(5.085 \%)$ \\
\hline Insertion bases & $43,915(3.928 \%)$ & $36,001(3.374 \%)$ & $34,884(3.232 \%)$ \\
\hline
\end{tabular}

Table 4. Read length distribution of PacBio and ONT identified transcripts

\begin{tabular}{|c|c|c|c|}
\hline \multirow{2}{*}{ Sequencing type } & \multicolumn{3}{|c|}{ Read length } \\
\cline { 2 - 4 } & Mean & median & maximum \\
\hline PacBio & 2072.57 & 1988 & 8105 \\
\hline ONT Dc & 1249.97 & 1135 & 6763 \\
\hline ONT Pc & 1474.28 & 1332 & 7319 \\
\hline
\end{tabular}

\section{Figures}

Figure 1

Classification of ROls. 

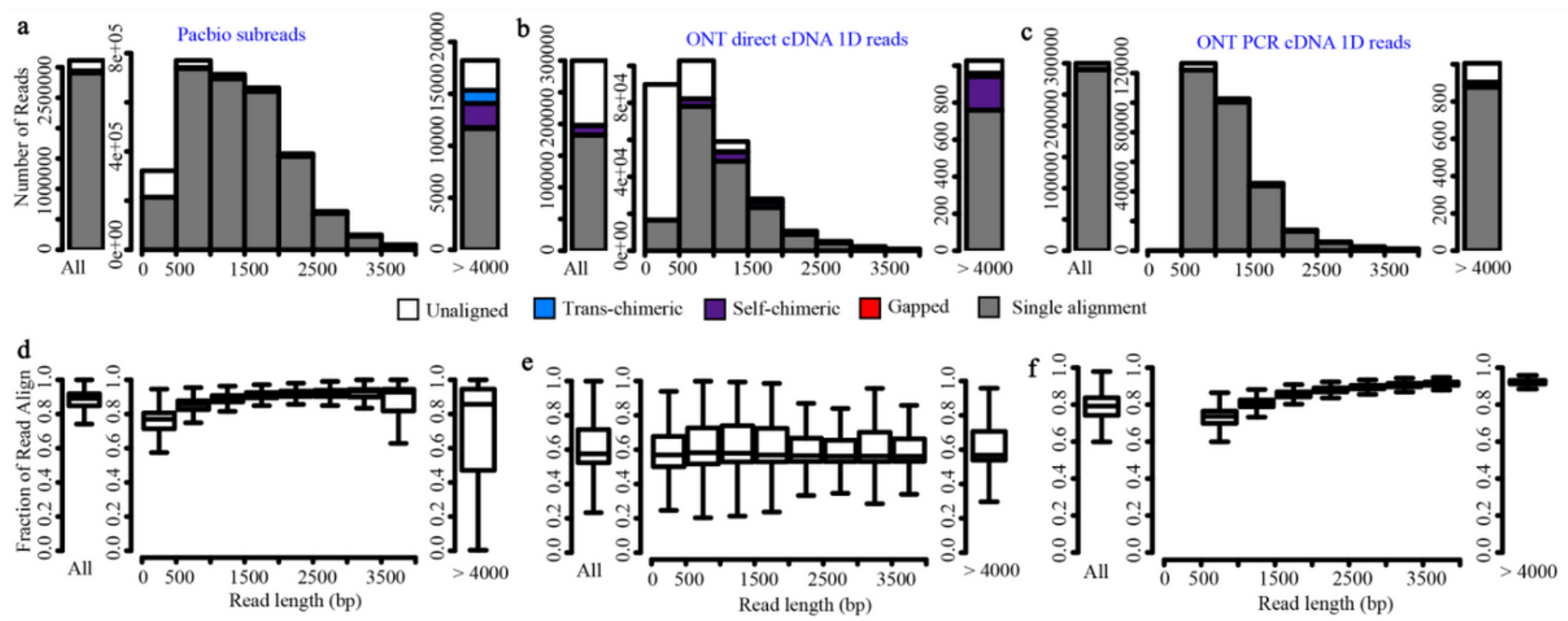

\section{Figure 2}

Length distributions and mappability of reads. a-c the length distributions of PacBio subreads (a), ONT Dc 1D reads (b), and ONT Pc 1D reads (c). Aligned reads are colour coded to indicate the fractions of reads that are unaligned (white), trans-chimeric alignments (blue), self-chimeric alignments (purple), gapped alignments consisting of multiple paths (red), and single best alignments (grey). The leftmost bar represents all reads, the middle portion represents reads from 0 to $4 \mathrm{~kb}$ in length, and the rightmost represents reads $>4 \mathrm{~kb}$ in length. $d-f$ the mappability of different length bins of PacBio subreads (d), ONT Dc 1D reads (e), and ONT Pc 1D reads (f). The leftmost bar represents the fraction of the mappable read length of the total read length for all reads. The middle section shows the mappable fraction for read lengths ranging from 0 to $4 \mathrm{~kb}$ in 500-bp increments, and the rightmost bar represents the mappable fraction of reads $>4 \mathrm{~kb}$.

\section{Figure 3}

Context-specific errors. Context-specific errors of a PacBio subreads, b ONT Dc 1D reads, and c ONT Pc 1D reads. The error types shown are insertions, deletions, and mismatches. For insertions (deletions), the large base above the plot indicates the inserted (deleted) base. For mismatch errors, the large base to the left (above) indicates the expected (observed) base. Blocks of coloured tiles show the error frequency in specific contexts for each error; the small base to the left (above) indicates the base preceding (following) the error. Error frequency is plotted on separate scales for insertions, deletions, and mismatches.

\section{Figure 4}

Alternative splicing event distributions of a PacBio subreads, b ONT Dc 1D reads, and c ONT Pc 1 D reads. 


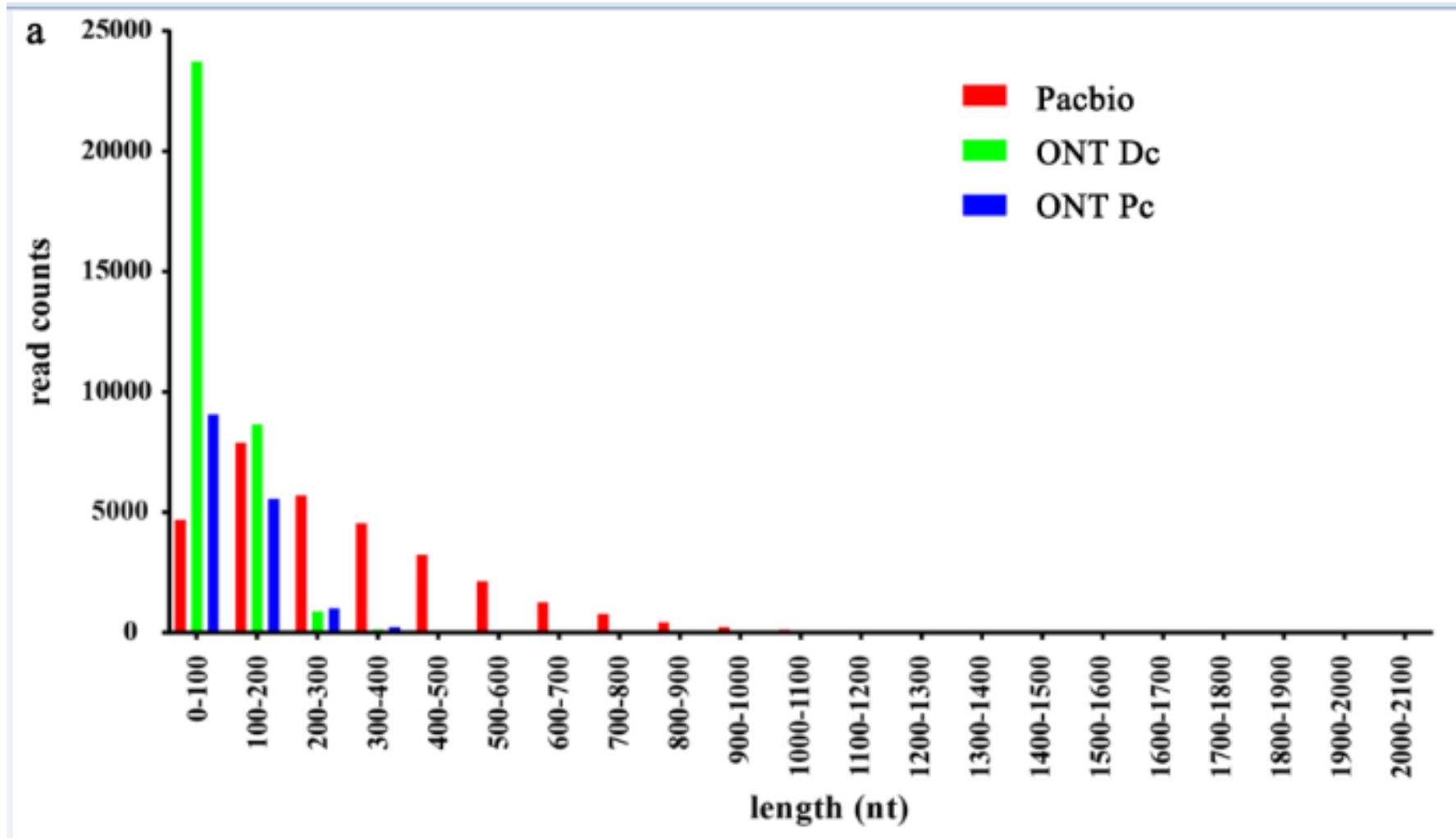

b

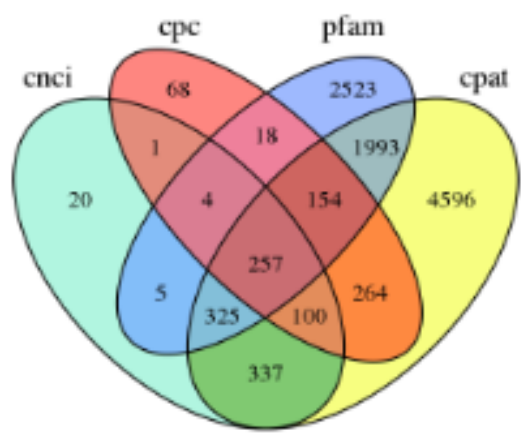

c ONT De

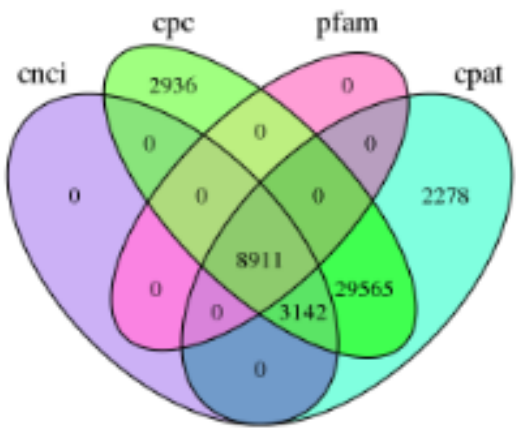

d ONT Pc

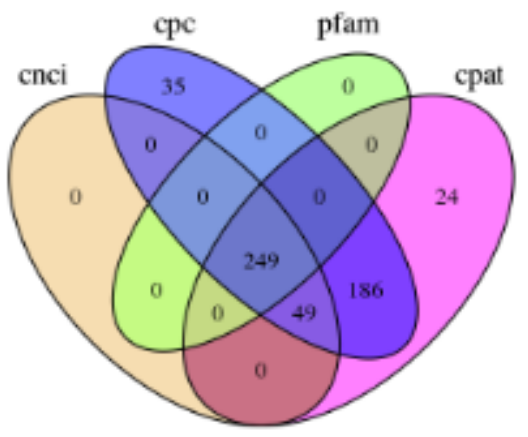

Figure 5

Length distributions of complete ORFs and identification of IncRNAs. a length distributions of the CDS lengths of complete ORFs in PacBio, ONT Dc, and ONT Pc results, b-d venn diagrams showing the numbers of candidate IncRNAs screened using Pfam, CPC, CNCl, and CPAT for PacBio (b), ONT Dc (c), and ONT Pc (d) results.

展

\section{Figure 6}

Sequence validation of predicted IncRNAs. a PCR products of randomly selected IncRNAs, b alignment between Sanger sequencing results and PacBio sequencing results for identified IncRNAs. c Alignment between Sanger sequencing results and ONT Pc sequencing results for identified IncRNAs. 


\section{Figure 7}

Transcript expression correlations between Illumina and ONT results. a-c expression correlations between Illumina and ONT Dc results in CTRL1, CTRL2, and CTRL3. $d-f$ expression correlations between Illumina and ONT Pc results in CTRL1, CTRL2, and CTRL3.

\section{Supplementary Files}

This is a list of supplementary files associated with this preprint. Click to download.

- TableS3.Pacbiosequencingresults.docx

- TableS1.Summaryofcleandata.docx

- TableS7.PrimersequencesforPCRvalidation.docx

- TableS5.SSRidentificationofPacbioandONTdata.docx

- Tables2.Illuminareadsmappedtothereferencegenome..docx

- TableS4.Nanoporesequencingresults.docx

- TableS6.CommonIncRNAsbetweenPacbioandONTPcdata.xlsx 